This item was submitted to Loughborough's Institutional Repository (https://dspace.lboro.ac.uk/) by the author and is made available under the following Creative Commons Licence conditions.

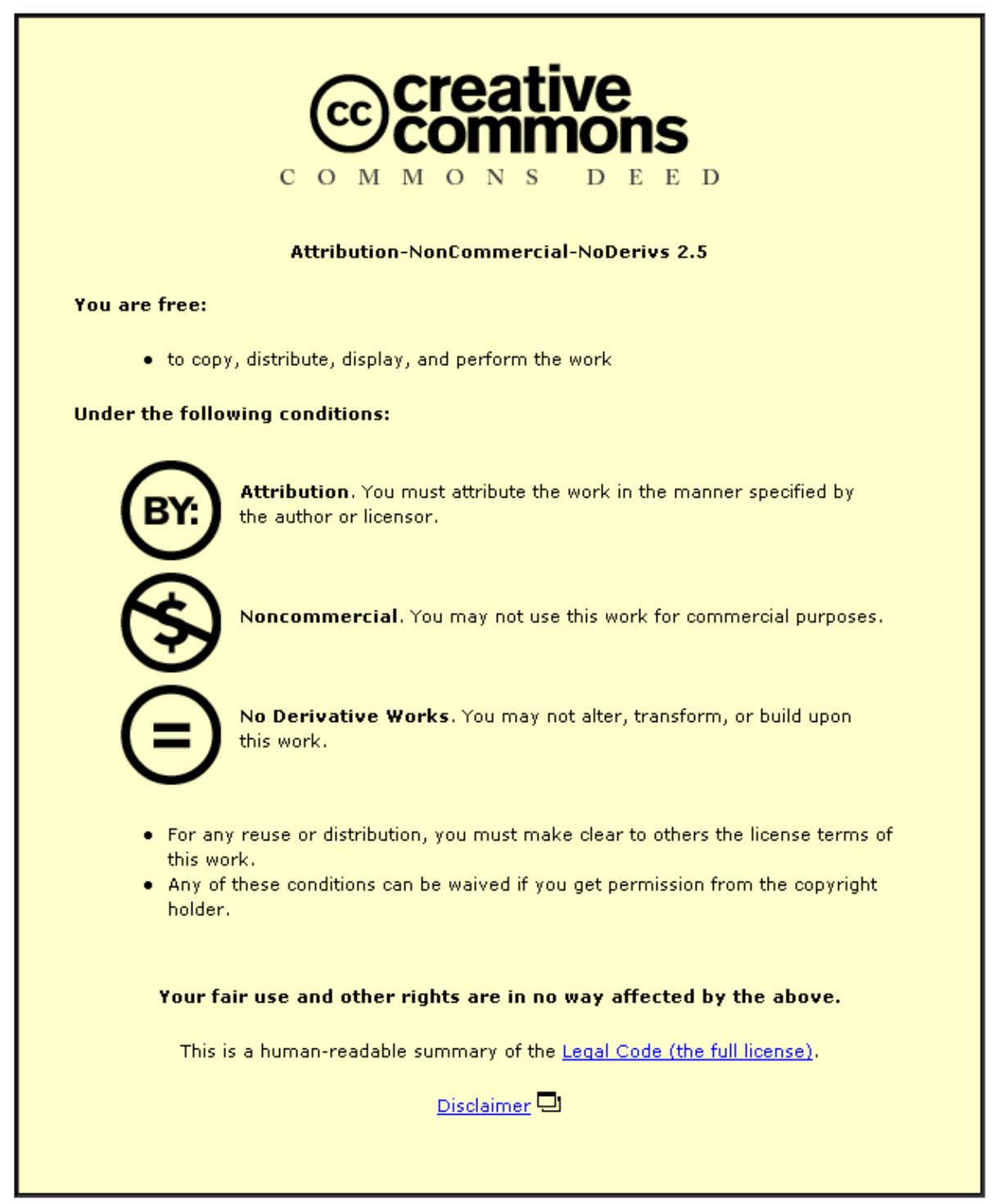

For the full text of this licence, please go to: http://creativecommons.org/licenses/by-nc-nd/2.5/ 


\title{
Singlet and triplet bipolarons on the triangular lattice
}

\author{
J.P.Hague ${ }^{\text {a }}$ P.E.Kornilovitch ${ }^{\text {b }}$ J.H.Samson ${ }^{\text {a }}$ A.S.Alexandrov ${ }^{\mathrm{a}}$ \\ a Department of Physics, Loughborough University, Loughborough, LE11 3TU, UK \\ ${ }^{\mathrm{b}}$ Hewlett-Packard Company, 1000 NE Circle Blvd, Corvallis, Oregon 97330, USA
}

\begin{abstract}
We study the Coulomb-Fröhlich model on a triangular lattice, looking in particular at states with angular momentum. We examine a simplified model of crab bipolarons with angular momentum by projecting onto the low energy subspace of the Coulomb-Fröhlich model with large phonon frequency. Such a projection is consistent with large long-range electron-phonon coupling and large repulsive Hubbard $U$. Significant differences are found between the band structure of singlet and triplet states: The triplet state (which has a flat band) is found to be significantly heavier than the singlet state (which has mass similar to the polaron). We test whether the heavier triplet states persist to lower electron-phonon coupling using continuous time quantum Monte Carlo (QMC) simulation. The triplet state is both heavier and larger, demonstrating that the heavier mass is due to quantum interference effects on the motion. We also find that retardation effects reduce the differences between singlet and triplet states, since they reintroduce second order terms in the hopping into the inverse effective mass. PUBLISHED AS: Journal of Physics and Chemistry of Solids Volume 69, Issue 12, December 2008, Pages 3304-3306
\end{abstract}

Key words: Bipolarons, electron-phonon interactions, unconventional superconductivity

\section{Introduction}

The possible role of local pairs in the cuprates and the origin of the pairing mechanism are subject to intense debate in the superconductivity community. Many experiments now demonstrate that there is a strong electron-phonon (e-ph) interaction in the cuprates. In particular, isotope effects show that lattice vibrations do play a role in determining the specific electronic structure of high-temperature superconductors [1]. However, consensus has not yet been reached about the effects of lattice vibrations. Theorists proposing a view of intrinsic repulsionmediated superconductivity claim that the isotope effects are simply due to change of the polaron

\footnotetext{
* Tel: +44-1509-228208. Fax: +44-1509-223986

Email address: J.P.Hague@lboro.ac.uk (J.P.Hague).

1 We acknowledge support from EPSRC grants $\mathrm{EP} / \mathrm{C} 518365 / 1$ and EP/D07777X/1
}

mass, which are the underlying quasi-particles for their theories, but that otherwise the e-ph interaction does not lead to the pairing required for superconductivity [2]. Here, we explore a scenario in which the e-ph interaction is so strong that pairing is into local bipolarons, which form a Bose-Einstein Condensate with no electronic resistance [3].

The possibility of bipolaronic superconductors in real materials has been critisised on the grounds that, in the presence of strong Coulomb repulsion, the e-ph interaction would have to be huge to form bound pairs. Thus, individual polarons forming the bipolaron would be extremely heavy [4]. For strong e-ph coupling on rectangular lattices with only nearest-neighbour hopping, it can be demonstrated that bipolaron mass is proportional to polaron mass squared [5], indicating that bipolaronic superconductivity on such lattices could only be a low temperature phenomenon. However, a more 


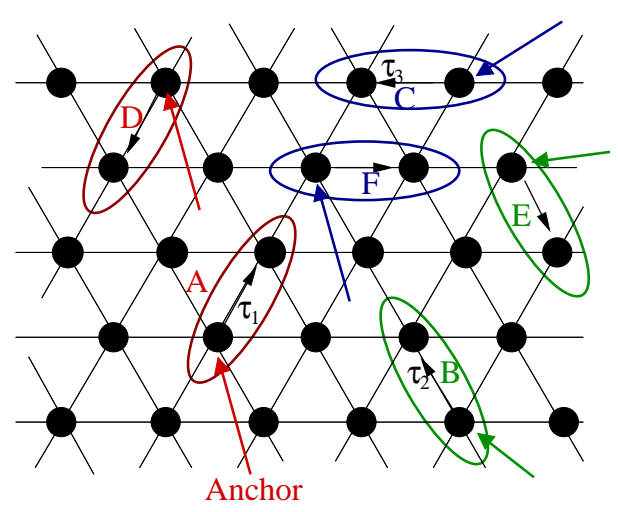

Fig. 1. (Colour online) Schematic of the small bipolaron on a triangular lattice with infinite Hubbard repulsion and long range attraction. For strong e-ph coupling, the energy space is such that bipolarons must be composed of dimers between nearest neighbour sites. There are six possible dimer configurations on the triangular lattice, categorised according to the relative positions of the first and second inserted particles. Configurations A and D etc. can be related through and exchange and translation. The most general 2 particle wavefunction is a linear combination of these six states, symmetrised according to translational (Bloch) and rotational (angular momentum) invarience.

recent scenario involves the possibility that e-ph interactions are longer range than the Coulomb repulsion [6], leading to inter-site pairs which can move freely on lattices formed from triangular plaquettes (crab bipolarons). Here, we consider the difference between singlet and triplet bipolarons on the triangular lattice. Given recent results concerning the possibility of $d$-wave superconductivity mediated via phonons [7], we consider this a first step towards understanding unconventional pairing on a quantitative footing using numerically exact QMC computations.

We study the Coulomb-Fröhlich model for longrange e-ph interactions in quasi-2D materials,

$$
\begin{aligned}
H & =-t \sum_{\left\langle\mathbf{n} \mathbf{n}^{\prime}\right\rangle \sigma} c_{\mathbf{n}^{\prime} \sigma}^{\dagger} c_{\mathbf{n} \sigma}+\sum_{\mathbf{n n}^{\prime} \sigma} V\left(\mathbf{n}, \mathbf{n}^{\prime}\right) c_{\mathbf{n} \sigma}^{\dagger} c_{\mathbf{n} \sigma} c_{\mathbf{n}^{\prime} \bar{\sigma}}^{\dagger} c_{\mathbf{n}^{\prime} \bar{\sigma}} \\
& +\sum_{\mathbf{m}} \frac{\hat{P}_{\mathbf{m}}^{2}}{2 M}+\sum_{\mathbf{m}} \frac{\xi_{\mathbf{m}}^{2} M \omega^{2}}{2}-\sum_{\mathbf{n} \mathbf{m} \sigma} f_{\mathbf{m}}(\mathbf{n}) c_{\mathbf{n} \sigma}^{\dagger} c_{\mathbf{n} \sigma} \xi_{\mathbf{m}} .
\end{aligned}
$$

$\xi_{\mathbf{m}}$ is the ion displacement, sites are numbered by $\mathbf{n}$ $/ \mathbf{m}$ for electrons / ions respectively and $c$ annihilate electrons. The phonons are independent oscillators with frequency $\omega$ and mass $M . \hat{P}_{\mathbf{m}}=-i \hbar \partial / \partial \xi_{\mathbf{m}}$ is the ion momentum operator. Since electrons are mobile in the plane, in-plane Coulomb repulsion $V\left(\mathbf{n}-\mathbf{n}^{\prime}\right)$ is heavily screened. We consider only infinite on site repulsion $U$. The Fröhlich interaction is specified via the force function, $f_{\mathbf{m}}(\mathbf{n})=$

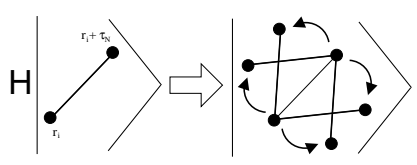

Fig. 2. Schematic of the hopping term as a set of rotations, showing that the hopping operator is also related to the angular momentum operator.

$\kappa\left[(\mathbf{m}-\mathbf{n})^{2}+1\right]^{-3 / 2}$, where $\kappa$ is a constant [8]. The dimensionless e-ph coupling $\lambda$ is defined as $\lambda=\sum_{\mathbf{m}} f_{\mathbf{m}}^{2}(0) / 2 M \omega^{2} z t$ ( $z$ is the coordination number). Long-range interactions make polarons light, and less dependent on lattice type $[8,9,10,11]$.

\section{Unconventional pairing and the crab bipolaron}

To obtain insight into the crab bipolaron, we consider pairs in the limit where on-site Coulomb repulsion is infinite, inter-site attraction is large and phonon frequency is large (anti-adiabatic limit $\omega>$ $z t \lambda$ ). Thus the lowest energy states span a subspace of near-neighbour pairs. The most general wavefunction in this basis is,

$$
|\psi\rangle=\frac{1}{N R} \sum_{\mathbf{r}_{i}} e^{i \mathbf{k} \cdot \mathbf{r}_{i}} \sum_{l=1}^{R} e^{\frac{i l m 2 \pi}{R}} \sum_{n=1}^{R} a_{n} A_{\mathbf{r}_{i}}^{(n+l) \dagger}|0\rangle
$$

that is that Bloch's theorem and the equivalent for rotated angular momentum states have been applied to a linear combination of all neighbour pairs. $R$ is the order of rotational symmetry, $m$ is the angular momentum. $A_{\mathbf{r}_{i}}^{(N) \dagger}=c_{\mathbf{r}_{i}}^{\dagger} c_{\mathbf{r}_{i}+\tau_{N}}^{\dagger}$ and $\tau_{N}$ are the nearneighbour vectors.

There are a number of symmetries that can be used to simplify this expression: $A_{\mathbf{r}_{i}}^{(N+R / 2) \dagger}=$ $-A_{\mathbf{r}_{i}-\tau_{N}}^{(N) \dagger}, A_{\mathbf{r}_{i}}^{(N+R) \dagger}=A_{\mathbf{r}_{i}}^{(N) \dagger}$, making the substitution $\bar{a}_{n}=a_{n}+(-1)^{m} a_{n+R / 2}$ and noting that $e^{2 \pi i m(l+R / 2) / R}=(-1)^{m} e^{\frac{i l m 2 \pi}{R}}$, the wavefunction can be simplified to:

$$
\begin{aligned}
|\psi\rangle= & \frac{1}{N R} \sum_{\mathbf{r}_{i}} e^{i \mathbf{k} \cdot \mathbf{r}_{i}} \sum_{l=1}^{R / 2} e^{\frac{i l m 2 \pi}{R}} \\
& \times \sum_{n=1}^{R / 2} \bar{a}_{n-l}\left(A_{\mathbf{r}_{i}}^{(n) \dagger}+(-1)^{m} A_{\mathbf{r}_{i}}^{(n+R / 2) \dagger}\right)|0\rangle
\end{aligned}
$$

i.e. the problem is reformulated as $R / 2$ singlet and $R / 2$ triplet bands.

The effect of the hopping term on pairs can be written as a rotation, showing that hopping also has 

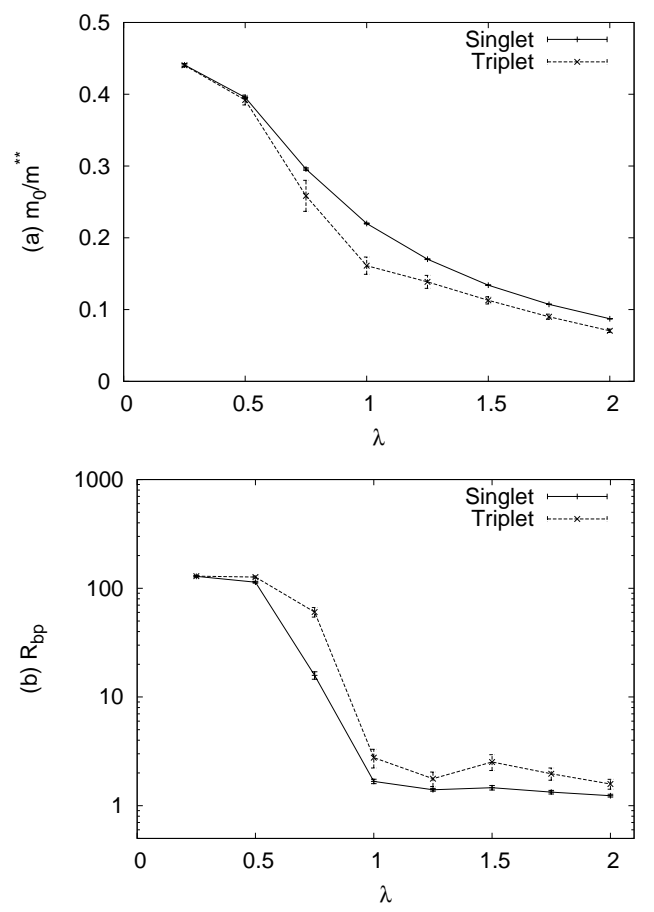

Fig. 3. Singlet and triplet states on a triangular lattice with infinite Coulomb repulsion. $\bar{\beta}=3.5$. Paths are constrained to be within 200 lattice spacings of each other. Measurements were taken every $10 \mathrm{MC}$ steps, with error bars computed by blocking groups of 1000 measurements. Results are computed for $\bar{\omega}=4$. Panel (a) shows the inverse masses of singlet and triplet bipolarons, and panel (b) their radii. The masses of the triplet bipolarons are generally larger than their singlet counterparts. The radii of triplet bipolarons are also larger, as should be expected due to the node in the wavefunction. However, this makes the heavier mass more surprising, since larger (i.e. less well bound) bipolarons would be expected to be lighter. The apparent convergence of the singlet and triplet properties at large $\lambda$ is due to retardation effects.

some of the properties of an angular momentum operator (see figure 2).

$$
\begin{aligned}
\tilde{H}_{t b} A_{\mathbf{r}_{i}}^{(N) \dagger}=-\tilde{t} & \left(A_{\mathbf{r}_{i}}^{(N+1) \dagger}+A_{\mathbf{r}_{i}}^{(N-1) \dagger}\right. \\
& \left.-A_{\mathbf{r}_{i}+\tau_{N}}^{(N+1+R / 2) \dagger}-A_{\mathbf{r}_{i}+\tau_{N}}^{(N-1+R / 2) \dagger}\right)
\end{aligned}
$$

If the basis didn't just contain nearest neighbour pairs, then $\tilde{H}_{t b}$ would introduce $m$ dependent functions into the result. However for only nearest neighbour pairs, eq. 2 can be rewritten as,

$|\psi\rangle=\frac{1}{N R} \sum_{\mathbf{r}_{i}} e^{i \mathbf{k} \cdot \mathbf{r}_{i}} \sum_{l=1}^{R / 2} e^{\frac{i l m 2 \pi}{R}} \sum_{n=1}^{R / 2} \bar{a}_{n-l} \bar{A}_{\mathbf{r}_{i}}^{(n) \dagger}|0\rangle$

where, $\bar{A}_{\mathbf{r}_{i}}^{(n) \dagger}=A_{\mathbf{r}_{i}}^{(n) \dagger}+(-1)^{m} A_{\mathbf{r}_{i}}^{(n+R / 2) \dagger}$ and $\bar{a}_{n}=$ $a_{n}+(-1)^{m} a_{n+R / 2}$. Thus, there is no dependence on angular momentum. $s, d$ etc. states are degenerate singlets, $p, f$ etc. are degenerate triplets. Initially, the degeneracy of the angular momentum states seems surprising. However, since only the near-neighbour subspace is considered, there are only $6 \mathrm{~N}$ degrees of freedom. As an increasing number of possible pairing arrangements is considered (for example if $U$ were not infinite), the total number of degrees of freedom would increase, and the degeneracy would be lifted.

As we have previously discussed, singlet and triplet states can be mapped onto a dimer lattice, with hopping $-\tilde{t}$ for singlets and $\tilde{t}$ for triplets (the difference in hopping sign can be demonstrated by considering only two particles on a singlet trianglular molecule, symmetrised according to eq. 2) [12]. Thus one can determine the secular equations:

$E\langle N \mid \psi\rangle=\langle N|\tilde{H}| \psi\rangle$

where $|N\rangle=\bar{A}^{(N) \dagger}|0\rangle$. The solution to those equations is given by the equation,

$\operatorname{det}\left|H_{\mathbf{k}}-E\right|=\mp \tilde{t}\left|\begin{array}{ccc} \pm E / \tilde{t} & 1+\gamma_{\mathbf{k}}^{*} & 1+\beta_{\mathbf{k}} \\ 1+\gamma_{\mathbf{k}} & \pm E / \tilde{t} & 1+\alpha_{\mathbf{k}}^{*} \\ 1+\beta_{\mathbf{k}}^{*} & 1+\alpha_{\mathbf{k}} & \pm E / \tilde{t}\end{array}\right|=0$

with $\left.\alpha_{\mathbf{k}}=\exp \left(-i a k_{x} / 2+i \sqrt{3} a k_{y} / 2\right)\right), \quad \beta_{\mathbf{k}}=$ $\exp \left(-i k_{x} a / 2-i \sqrt{3} k_{y} a / 2\right)$ and $\gamma_{\mathbf{k}}=\exp \left(i k_{x} a\right)$.

There are 6 bands resulting from the diagonalisation of the Hamiltonian. Three singlet (corresponding to even $m$ ) and three triplet (odd $m$ states),

$$
\begin{aligned}
& E_{1}(\mathbf{k})=V_{\min } \pm \tilde{t}(-1-\sqrt{3+\epsilon(\mathbf{k}) / \tilde{t}}) \\
& E_{2}(\mathbf{k})=V_{\min } \pm \tilde{t}(-1+\sqrt{3+\epsilon(\mathbf{k}) / \tilde{t}}) \\
& E_{3}(\mathbf{k})=V_{\min } \pm 2 \tilde{t}
\end{aligned}
$$

where $\epsilon(\mathbf{k})=-2 \tilde{t}\left(\cos k_{x} a+\cos \left(k_{x} a / 2-\sqrt{3} k_{y} a / 2\right)+\right.$ $\left.\cos \left(k_{x} a / 2+\sqrt{3} k_{y} a / 2\right)\right)$ is the polaron band structure in the antiadiabatic limit. An unusual result is that the singlet bipolaron mass is proportional to the polaron mass, $m_{s}^{* *}=6 m^{*}$ while the triplet bipolaron mass is infinite. In the rest of this article, we compute singlet and triplet states computed using a continuous time QMC algorithm to determine which of these attributes survive into the intermediate coupling and phonon frequency regime $[13,12]$. 


\section{Quantum Monte-Carlo results}

QMC simulations of the crab bipolaron state have been carried out for singlet states [13,12]. We have also simulated triplet bipolarons on the chain [14]. It is of interest to determine if the limiting analytic results discussed in the previous section can be realised in numerical simulations. We have previously determined that there are significant differences between the masses of bipolarons on staggered and rectangular ladders $[12,5]$. We have also found that masses of polarons and bipolarons on lattices with triangular components have similar magnitude [13].

We compute numerical results from the continuous time QMC algorithm for a bipolaron on a triangular lattice. The singlet and triplet properties are computed by symmetrising the waveunctions. So the triplet bipolaron picks up a sign change on exchange, whereas the singlet always has positive sign. This affects the estimators, which depend on the sign as,

$R_{b p}=\left\langle s \sqrt{\frac{1}{\beta} \int_{0}^{\beta}\left(\mathbf{r}_{1}(\tau)-\mathbf{r}_{2}(\tau)\right)^{2} d \tau}\right\rangle$

$\frac{m_{0}}{m_{i}^{* *}}=\lim _{\beta \rightarrow \infty} \frac{1}{\bar{\beta}}\left\langle s \Delta \mathbf{r}_{i}^{2}\right\rangle$

For configurations where the end points of the paths are together, $s=0 . \Delta \mathbf{r}$ is defined as in ref. [9].

Figure 3 shows the effective mass and size of singlet and triplet states on a triangular lattice with infinite local Coulomb repulsion. Results are computed for $\omega / t=4$. Panel (a) shows the inverse masses of singlet and triplet bipolarons, and panel (b) their radii. It can be seen that the masses of the triplet bipolarons are generally larger than their singlet counterparts. The radii of triplet bipolarons are also larger, as should be expected due to the node in the wavefunction. However, this makes the heavier mass more surprising, since larger bipolarons would be expected to be lighter since they are less well bound. The larger mass is thus due to quantum interference effects in the motion. This can be best understood by imagining the tight binding model constructed for hopping of bipolarons. The bipolaron overlap integrals giving the effective hopping are highly sensitive to the form of the binding wavefunction, and it is clear that the node in the triplet wavefunction will make those integrals (i.e. the effective hopping) much smaller leading to smaller effective mass. For larger couplings, zt $\lambda>\omega$, the antia- diabatic approximation used in the previous section does not hold. Thus the largest differences between triplet and singlet states are found around $\lambda=1$.

The apparent convergence of singlet and triplet states at large coupling is initially surprising. However, analysis of the sign can shed light on this effect. For weak coupling, any bipolarons are weakly bound, so singlet-triplet splitting must be very small, since it can't be larger than the singlet binding energy. Since $\Delta E_{s t}=-(\ln \langle s\rangle) / \beta$, the average sign must be approximately 1 . At large coupling, paths must be nearly straight at very strong coupling due to retardation effects. Since there is infinite Coulomb repulsion, at least two kink insertions are required on each path for an exchange, exchanges become increasingly rare, and the sign must return to 1 . So the total sign dips (and thus the difference in mass is largest) when $z t \lambda \sim \omega$.

\section{Conclusion}

We have computed properties of bipolarons with angular momentum on triangular lattices. The mass of the singlet bipolaron is of the order of the polaron mass for a wide range of couplings. However, we find that triplet bipolarons have much larger mass for coupling $z t \lambda \sim \omega$. A surprising result here is that retardation effects at very large $\lambda$ reduce the difference between singlet and triplet properties when the local Coulomb repulsion is infinite. Understanding of the full range of bipolaron behaviours, especially on unconventional lattices is clearly important, and will form the subject of future publications.

\section{References}

[1] G. Zhao, D. E. Morris, Phys. Rev. B 51 (1995) 16487.

[2] P. W. Anderson, P. A. Lee, M. Randeria, T. M. Rice, N. Trivedi, F. C. Zhang, J. Phys.: Condens. Matter 16 (2004) R755.

[3] A. S. Alexandrov, J. Ranninger, Phys. Rev. B 23 (1981) 1796.

[4] E. V. L. de Mello, J. Ranninger 58 (1998) 9098.

[5] J. P. Hague, P. E. Kornilovitch, J. H. Samson, A. S. Alexandrov, J. Phys.: Conf. Series(Submitted for proceedings of Phonons 2007).

[6] A. S. Alexandrov, Phys. Rev. B 53 (1996) 2863.

[7] J. P. Hague, Phys. Rev. B 73 (2006) 060503(R).

[8] A. S. Alexandrov, P. E. Kornilovitch, Phys. Rev. Lett. 82 (1999) 807.

[9] P. E. Kornilovitch, Phys. Rev. Lett 81 (1998) 5382.

[10] P. E. Kornilovitch, Phys. Rev. B 60 (1999) 5382. 
[11] J. P. Hague, P. E. Kornilovitch, A. S. Alexandrov, J. H. Samson, Phys. Rev. B 73 (2006) 054303.

[12] J. P. Hague, P. E. Kornilovitch, J. H. Samson, A. S. Alexandrov, J. Phys.: Condens. Matter 19 (2007) 255214.

[13] J. P. Hague, P. E. Kornilovitch, J. H. Samson, A. S. Alexandrov, Phys. Rev. Lett. 98 (2007) 037002.

[14] J. P. Hague, P. E. Kornilovitch, A. S. Alexandrov, J. H. Samson, Physica C 460-462 (2007) 1121-1122. 